CASO CLÍNICO

\title{
Granuloma anular en niños: serie de casos
}

\author{
Annulare Granuloma in children: case series
}

Gustavo A. Lizardo Castro, ${ }^{1}$ Aleydi Gómez Campos. ${ }^{2}$

'Pediatra, Sub-especialista en Dermatología Pediátrica, Departamento de Pediatría, Servicio de Dermatología, Hospital Escuela; Facultad de Ciencias Médicas (FCM), UNAH;

2Médica Especialista en Dermatología, egresada de FCM, UNAH, Tegucigalpa.

RESUMEN. Antecedentes: El Granuloma anular es una dermatosis benigna, autolimitada, de etiología desconocida, más frecuente en el género femenino y caracterizada por pápulas que adoptan usualmente una configuración anular. De acuerdo a su presentación ha sido clasificado en: localizado, generalizado, subcutáneo y perforante. El diagnóstico es clínico, pero en casos dudosos se recurre a estudios histopatológicos y dermatoscópicos. Su curso es generalmente autolimitado por lo que una conducta expectante, es lo más apropiado. Descripción de casos clínicos: Se presentan 3 casos pediátricos evaluados en la consulta externa del servicio de dermatología del Hospital Escuela, por lesiones localizadas principalmente en manos y pies. El caso 1 y 2, corresponden típicamente a granuloma anular localizado. El caso 3 presentó lesiones poco usuales, tipo pápulas de 1 a $3 \mathrm{~mm}$ de diámetro con umbilicación central, correspondiendo clínicamente a una variante rara, de granuloma anular papular umbilicado. La biopsia confirmó el diagnóstico de granuloma anular en los 3 casos. Conclusiones: El conocimiento de esta patología relativamente frecuente, permitirá al gremio médico en general y pediatras en particular, reconocer que se trata de una enfermedad que tiene un curso benigno y autolimitado, evitando así procedimientos agresivos e innecesarios.

Palabras clave: Enfermedades de la piel, Granuloma anular, Niño.
Recibido: 08-11-2019 Aceptado para publicación 05-01-2021

Dirección para correspondencia: Dr. Gustavo Lizardo

Correo electrónico: glizardoc@yahoo.com

Declaración de relaciones y actividades financieras y no financieras y conflictos de interés: ninguno.

\section{INTRODUCCIÓN}

Granuloma anular (GA) es una dermatosis inflamatoria, benigna, de etiología desconocida, descrita por Colcott Fox en 1895. Radcliffe-Crocker en 1902 propusieron el nombre de granuloma anular. ${ }^{1-3}$ Su curso es autolimitado y en el $75 \%$ de los pacientes remite en 2 años, siendo frecuente las recurrencias $(40 \%)$ pero igualmente involucionan. ${ }^{4}$

El GA está caracterizado por pápulas que a menudo asumen una configuración anular. ${ }^{5}$ Desde el punto de vista clínico el granuloma anular se ha clasificado en localizado, generalizado, subcutáneo y perforante, ${ }^{2-5}$ sin embargo, hay varios reportes en la literatura de otras variantes clínicas: macular, lineal, papular, papular umbilicado y en parche.

El propósito de este artículo es presentar tres casos de granuloma anular. Dos de los casos, con la forma localizada y uno con la rara variante conocida como papular umbilicado. Todos los casos fueron atendidos en el servicio de dermatología del Hospital Escuela (H.E) en Tegucigalpa.

\section{Caso 1}

Niña de 10 años de edad, procedente del Distrito Central, sin antecedentes patológicos personales ni familiares de importancia. Acudió al servicio de dermatología H.E con dermatosis de 6 meses de evolución localizada en dorso de ambos pies, caracterizada por pápulas cupuliformes no descamativas, eritemato-violáceas, coalescentes formando una configuración aiiular, con involución central, levemente pruriginosa (Fig.1). Con diagnóstico clínico de granuloma anular se realizó biopsia de piel (Fig. 4A y 4B), que confirmó el diagnóstico.

\section{Caso 2}

Niño de 9 años de edad, procedente del Distrito Central, sin antecedentes de importancia. Fue referido a la Consulta Externa de Dermatología H.E por presentar dermatosis de 2 años de evolución que inició en dorso de manos y posteriormente en pies, caracterizada por pápulas cupuliformes no descamativas de color eritemato-violáceo, coalescentes formando una configuración anular, con involución central (Fig. 2). La biopsia de piel reportó granuloma necrobiótico intersticial consistente con granuloma anular. 


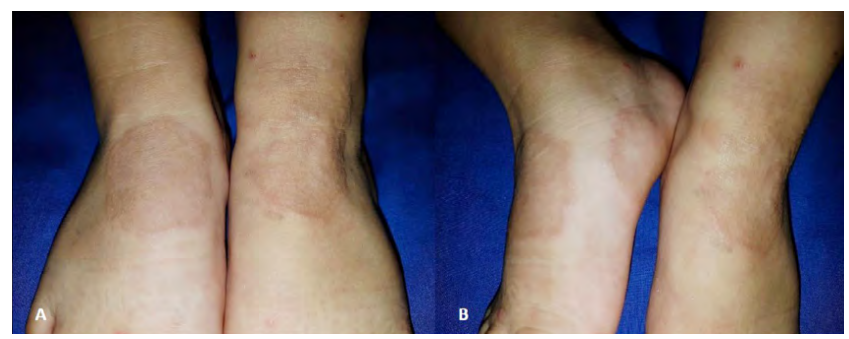

Figura 1. Granuloma anular localizado (caso 1). Lesiones en pies, vista frontal (A) y lateral (B): papulares en configuración anular, borde elevado, centro aclarado, sin presencia de escamas.

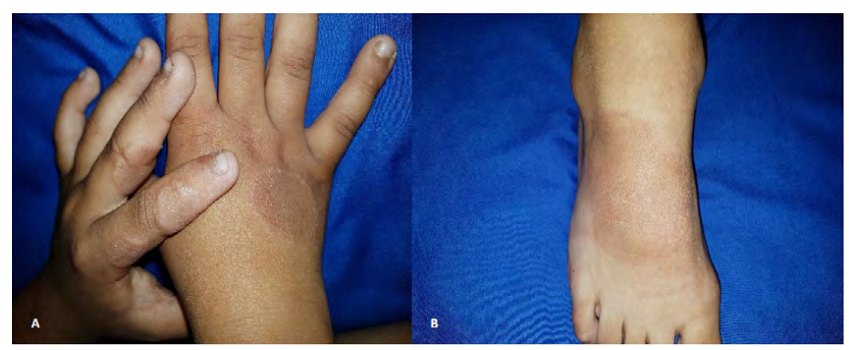

Figura 2. Granuloma anular localizado (caso 2). Lesiones en dorso de manos (A) y pies (B): papulares, configuración anular, borde elevado, centro aclarado, sin presencia de escamas.

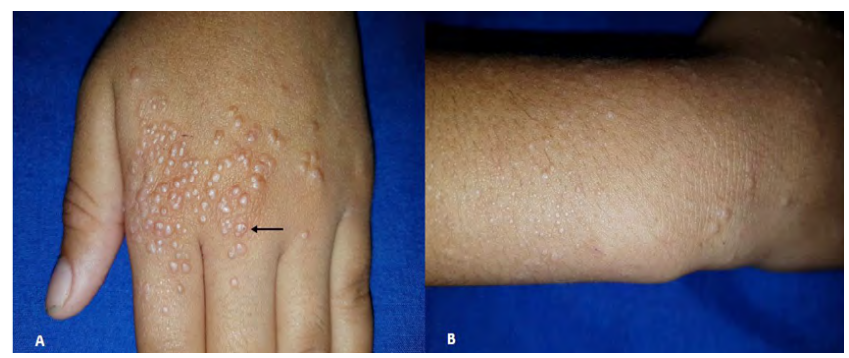

Figura 3. Granuloma anular papular umbilicado (caso 3). (A) Múltiples lesiones papulares color piel, sólidas, algunas con umbilicación central localizadas en el dorso de manos (flecha). (B) Lesiones papulares hipopigmentadas, que simulan un liquen nítido.

\section{Caso 3}

Niña de 8 años de edad, procedente del Distrito Central, sin antecedentes de importancia. Acudió al Servicio de Dermatología $\mathrm{HE}$, por presentar dermatosis de 21 meses de evolución, localizada en dorso de manos, región extensora de antebrazos, codos y escasas en rodillas, caracterizada por pápulas de 1 a $3 \mathrm{~mm}$ de diámetro, color piel, superficie cupuliforme, algunas con umbilicación central, asintomáticas (Fig. 3). Histopatología reportó granuloma necrobiótico intersticial, que en correlación clínica es consistente con granuloma anular papular (Fig. 4C y 4D).

\section{DISCUSIÓN}

El GA es una dermatosis relativamente frecuente en niños y adolescentes, de prevalencia incierta, más frecuente en el género femenino en relación 2:1, con diferentes variantes clínicas e histopatológicas, de etiología desconocida, pero que se ha asociado a infecciones cutáneas, luz ultravioleta, picadura

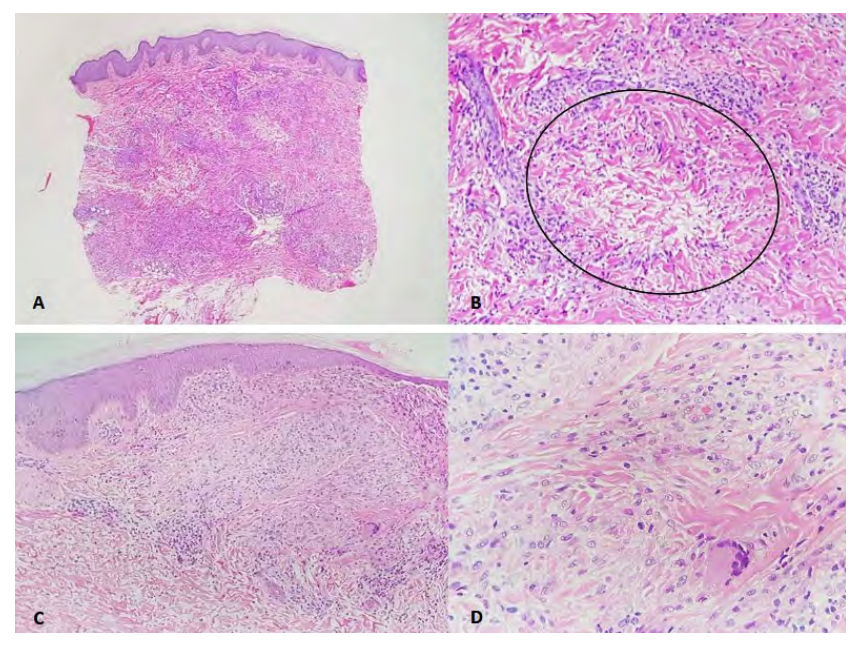

Figura 4. Imágenes histopatológicas (Hematoxilina-Eosina, H\&E) (caso 1). (A) Estrato córneo laminar con leve hiperqueratosis, estrato de Malpighi revela discreta acantosis(10x). (B) Necrobiosis incompleta en dermis, rodeada por linfocitos, histiocitos, muchos de ellos epitelioides que forman una empalizada parcial (óvalo), además pocas células gigantes multinucleadas tipo cuerpo extraño y Langhans (40x). (caso 3). (C) La epidermis presenta estrato córneo laminar ortoqueratósico con leve hiperqueratosis, estrato de Malpighi revela leve acantosis irregular y en una zona aplanamiento de los procesos interpapilares(10x). (D) Dermis papilar y reticular media exhiben área de necrobiosis incompleta rodeada por infiltrado inflamatorio compuesto por linfocitos, histiocitos, muchos de ellos epitelioides, con pocas células gigantes multinucleadas de tipo cuerpo extraño y Langhans (40x).

de insectos, traumatismo, tiroiditis autoinmune, diabetes, entre otras. ${ }^{1-6}$ Existen informes de GA después del uso de medicamentos tales como el oro, alopurinol, diclofenaco, quinidina, calcitonina, amlodipina y los inhibidores de la enzima convertidora de la angiotensina. ${ }^{7}$ Los intentos por identificar un subtipo de antígeno leucocitario humano (HLA) asociado, han mostrado resultados dispares en grupos de población distintos. Sin embargo, se ha podido determinar la asociación del GA localizado con HLA-A29 y GA generalizado con el HLA-A31 y HLA-Bw35. ${ }^{8}$ La patogenia de la enfermedad sigue siendo poco clara, existiendo las siguientes teorías: a) un proceso degenerativo del tejido conectivo como inicio de la inflamación granulomatosa, b) una reacción inmunitaria mediada por linfocitos, que resulta en la activación de macrófagos, con degradación de tejido conectivo mediado por citocinas $\mathrm{y}$, por último, c) una vasculitis sutil o microangiopatía que lleva a lesión tisular. ${ }^{7}$

EI GA localizado es la forma más frecuente manifestándose usualmente como una lesión anular o arciforme, color piel, eritematosa o violácea que mide de $1-5 \mathrm{~cm}$ de diámetro, borde elevado constituido por pápulas coalescentes, no descamativas firmes a la palpación y asintomáticas, como el caso 1 y el caso 2. Los sitios más afectados suelen ser el dorso de manos y pies, tobillos, miembros inferiores y muñecas, ${ }^{2}$ como ocurrió con los tres casos descritos. La variante perforante del GA se caracteriza por presentar múltiples pápulas umbilicadas, costra central o centro hiperqueratósico, las cuales pueden formar pústulas y ulcerarse, son pruriginosas y las observamos comúnmente en dorso de manos. Esta variante de GA en habitantes de Hawai tiene una incidencia del $5 \% .{ }^{8}$ Sin embargo se han descrito for- 
mas clínicas papulares umbilicadas mal definidas conocido como GA papular umbilicado como el caso 3 el cual presentó varias lesiones umbilicadas sobre todo en dorso de la mano izquierda (Fig. 3A) que según algunos autores representan un subtipo de la variante perforante, descrito por Lucky et al. en 1992, quienes consideran que la umbilicación central puede ser el resultado de la degeneración focal del colágeno; sin embargo, en la biopsia no suele encontrarse una perforación del tejido conectivo degenerado. Por este motivo se ha propuesto que es la forma intermedia entre el GA papular y el GA perforante, correspondiendo a una rara variante, habiéndose descrito para el 2011, ocho casos en la literatura anglosajona. ${ }^{9}$ El resto de lesiones puntiformes, hipocrómicas y no umbilicadas del caso 3 clínicamente semejan un liquen nítido (Fig. 3B).

El GA subcutáneo es una variante poco común y se presenta casi exclusivamente en niños entre 2 y 5 años de edad con predominio en el género femenino. Se caracteriza clínicamente por nódulos de $6 \mathrm{~mm}$ a $3,5 \mathrm{~cm}$ de diámetro, de consistencia firme, a menudo asintomáticos, localizados en dermis profunda e hipodermis. Los sitios mayormente afectados son las extremidades inferiores, especialmente el área pretibial, seguido por glúteos, manos y cuero cabelludo. ${ }^{10} \mathrm{El}$ GA generalizado representa del $2,8 \%$ al $15 \%$ de todos los casos, es más frecuente en adultos y se ha asociado con diabetes mellitus, trastornos del metabolismo de los lípidos, enfermedades malignas, infecciones como el virus de la hepatitis $\mathrm{C}$ y $\mathrm{B}, \mathrm{VIH}$, etc. Clínicamente se manifiesta con pápulas o nódulos, que pueden unirse y formar placas de 3-6 cm, que aumentan de forma centrífuga, anular, serpiginosa 0 arqueada. Las lesiones pueden afectar cualquier parte del cuerpo pero en general afectan el tronco y tienden a ser simétricas en las extremidades. ${ }^{11} \mathrm{La}$ variante parche de $\mathrm{GA}$, la forma más rara, se manifiesta por parches eritematosos o hiperpigmentados sin escama, asintomáticos, en el tronco y extremidades, predominando con mayor frecuencia en mujeres, puede adoptar una configuración anular y en la histopatología muestra los hallazgos clásicos de GA en empalizada o intersticial. ${ }^{12}$

El diagnóstico descansa sobre bases clínicas; sin embargo, en caso de duda la biopsia de piel confirmará el diagnóstico. La clasificación histopatológica se divide en granuloma clásico (en empalizada), como el caso 1 y granuloma intersticial, como el caso 2 y caso 3 . El granuloma clásico consiste en colágeno necrobiótico, fibrina y depósito de mucina, parcialmente rodeado por infiltrados de histiocitos y linfocitos, como lo reportado en caso 1 (Fig. 4A y 4B) y la forma Intersticial (difusa), en que la degeneración del colágeno es mínima y no se organiza en nódulos, afecta fibras aisladas aleatoriamente, que se observan hinchadas e intensamente eosinófilas, alternando con fibras aparentemente normales, rodeadas de histiocitos, las fibras se separan por depósitos de mucina, como el caso 3 (Fig. 4C y 4D). En el tipo perforante la eliminación transepidérmica con restos de necrobiosis están presentes próximos a la epidermis y se ven siendo engullidos por esta última, formando un canal perforante por el que el material necrótico es extruido a la superficie, a menudo, a través de un folículo piloso. ${ }^{8}$
La dermatoscopía es otra herramienta diagnóstica que revela vasos desenfocados los cuales presentan una morfología variable (punteado, lineal, irregular, ramificados) sobre un fondo de color rojizo. También suele observarse zonas focal o difusamente distribuidas con una coloración blanquecina o amarillonaranja que representan las características no vasculares más comunes del GA. Estos hallazgos característicos probablemente son resultado de la degeneración del colágeno y depósito de mucina. ${ }^{13} \mathrm{El}$ diagnóstico diferencial dependerá de la variante de GA según su clasificación, entre las entidades a descartar para el tipo localizado incluye: tiña del cuerpo, lupus eritematoso cutáneo subagudo, liquen plano anular, eritema migratorio cróni$\mathrm{Co}^{2}$ para el GA papular incluye: picaduras de insectos, urticaria papular, molusco contagioso, liquen nítido y entre las patologías a considerar en GA perforante se encuentran: varicela, sarcoidosis, erupciones a drogas, foliculitis perforante, pitiriasis liquenoide y varioliforme aguda, ${ }^{5}$ elastosis perforante serpiginosa y colagenosis perforante reactiva.

El tratamiento en general incluye resolución espontánea de las lesiones, en ocasiones la biopsia inicia este proceso. Los esteroides tópicos de alta potencia se han considerado tratamiento de primera línea, aunque su administración es limitada, por la extensión de las lesiones, apego al tratamiento y efectos secundarios. En cuanto a los esteroides sistémicos, se requieren dosis altas y usualmente los pacientes recidivan al omitirlos. Se han recomendado los inhibidores de la calcineurina, como tacrolimus y pimecrolimus tópico, debido a sus pocos efectos adversos y a la efectividad de los mismos. Los esteroides intralesionales a dosis $2.5 \mathrm{mg} / \mathrm{ml}$ también han sido eficaces. La criocirugía con nitrógeno líquido ha presentado resultados favorables aunque no se ha definido la frecuencia óptima de aplicación. La extirpación quirúrgica, se asocia con diversos grados de éxito en el granuloma subcutáneo. ${ }^{2}$ La fototerapia con psoralenos más radiación ultravioleta $A$ (PUVA) son terapias de segunda línea. Otros tratamientos incluyen administración de hidroxicloroquina, isotretinoína o dapsona, con resultados impredecibles. La vitamina $\mathrm{E}$ oral o tópica se considera un tratamiento seguro y sin eventos adversos conocidos. ${ }^{8}$

El pronóstico del GA por lo general es bueno. La variante localizada y en parche, son asintomáticas y autolimitadas: ${ }^{14,15}$ la mayoría de los pacientes tiende a la involución espontánea en aproximadamente dos años. El GA papular umbilicado puede ser autolimitado, o persistir por años. ${ }^{9}$ En el caso del GA perforante tiende a producir lesiones cicatriciales ${ }^{2,8}$ EI GA generalizado es más resistente a la terapia tópica y sistémica por lo que presenta una evolución más lenta. ${ }^{11}$ El granuloma anular subcutáneo tiende a la resolución espontánea, por lo que se sugiere vigilancia periódica, a menos que su localización afecte la función de un órgano, está indicado el manejo quirúrgico. ${ }^{10}$

\section{CONCLUSIÓN}

El GA es una patología relativamente frecuente en niños y adolescentes, de curso benigno y autolimitado. Fundamentado en estas características, en la mayoría de los casos la 
conducta a seguir es expectante. El gremio médico en general y pediatras en particular deben reconocer esta patología en su forma clásica y sus variantes clínicas para ofrecer un manejo adecuado y oportuno evitando procedimientos agresivos e innecesarios.

\section{CONTRIBUCIONES}

Ambos autores participaron de manera similar en el diseño y análisis de los casos clínicos, en la redacción del manuscrito, incorporaron las recomendaciones editoriales y aprobaron la versión final del artículo.

\section{REFERENCIAS}

1. De Aloe G, Risulo M, Sbano P, Pianigiani E, Fimiani M. Congenital subcutaneous granuloma annulare. Pediatr Dermatol. 2005;22(3):234-6.

2. Prendiville JS. Granuloma Anular. En: Goldsmith L, Katz S, Gilchrest B, Paller A, Leffell D, Wolff K. Fitzpatrick Dermatología en Medicina General. Argentina: Editorial Médica Panamerican;. 2012 p. 467-72.

3. Chiang K, Bhalla R, Mesinkovska NA, Piliang MP, Tamburro JE. Periocular granuloma annulare: A case report and review of literature. Pediatr Dermatol. 2014;31(6):722-5.

4. Wolff K, Johnson RA, Saavedra AP, Fitzpatrick Atlas de dermatología clínica. $7^{\text {a }}$. ed. México: McGrawHill. 2014. Sección Granuloma anular. p. 375376.

5. Choi JC, Bae JY, Cho S, Choi JH, Sung KJ, Moon KC, et al. Generalized perforating granuloma annulare in an infant. Pediatr Dermatol. 2003;20(2):131-3.

6. Fathi K, Harangi F, Kravjak A, Pinter A. Subcutaneous granuloma annulare of the penis associated with a urethral anomaly: case report and review of the literature. Pediatr Dermatol. 2014;31(4):e100-3.

7. Leung AK, Barankin B. An annular lesion on the elbow. Am Fam Physician. 2016;93(5):397-8.

8. Navarro-Hernández CA, Soto-ortiz JA, Solís-Ledesma G, Navarro-Jímenez BR. Granuloma anular: un reto dermatológico. Dermatol Rev Mex 2018;62(3):221-32.

9. Tobón MX, Tovar Á, Rodríguez G. Granuloma anular papular umbilicado en un adolescente. Rev Med. 2012;20(2):115

10. Reyes-Baraona F, Hasbún Acuña $P$, González S, Zegpi MS. Subcutaneous granuloma annulare: A case report. Rev Chil Pediatr. 2017;88(5):652-5.

11. Pătraşcu V, Giurcă $C$, Ciurea RN, Georgescu CV. Disseminated granuloma annulare: Study on eight cases. Rom J Morphol Embryol. 2013;54(2):32731

12. Anubhav G, Sushma G, Gupta LK, Khare AK, Asit M. Extensive patch type granulomas annulare : A rare case report. IJMSRP. 2014;1(1):23-5.

13. Errichetti E, Lallas A, Apalla Z, Di Stefani A, Stinco G. Dermoscopy of Granuloma Annulare: A Clinical and Histological Correlation Study. Dermatology. 2017;233(1):74-9.
14. Piette EW, Rosenbach M. Granuloma annulare. Clinical and histologic variants, epidemiology, and genetics. J Am Acad Dermatol. 2016;75:457-65.

15. Gomes RT, Ribeiro Balizardo JGS, Zelandi Filho C, Vasconcelos Schaefe L. Patch-type granuloma annulare: a case report. Med (Ribeirao Preto Online). [Internet] 2017[consultado 15 jun 2019];50(2):130-3. Disponible en: http://revista.fmrp.usp.br/2017/vol50n2/RC4-Patch-type-granulomaannulare-a\%20case-report.pdf

ABSTRACT. Backgroung: Granuloma annulare is a benign, self-limited dermatosis of unknown etiology, more frequent in women and characterized by papules that usually adopt an annular configuration. According to its presentation, it has been classified as: localized, generalized, subcutaneous and perforating. The diagnosis is clinical, but in doubtful cases, histopathological and dermoscopic studies are used. Its course is generally self-limited so expectant behavior is the most appropriate. Description of clinical cases: Three pediatric cases evaluated in the External Consultation of the Dermatology Service, Hospital Escuela, are presented, for lesions located mainly in the hands and feet. Cases 1 and 2 typically correspond to a localized granuloma annulare. Case 3 presented unusual lesions, papules of 1 to $3 \mathrm{~mm}$ diameter with central umbilication, clinically corresponding to a rare variant of papular umbilicated granuloma annular. Biopsy confirmed the diagnosis of granuloma annulare in all 3 cases. Conclusions: Knowledge of this relatively frequent pathology will allow the medical profession in general and pediatricians in particular to recognize that it is a disease that has a benign and self-limiting course, thus avoiding aggressive and unnecessary procedures.

Keywords: Child, Granuloma annulare, Skin diseases. 